\title{
A Model for Hyphal Growth and Branching
}

\author{
By J. I. PROSSER* \\ Department of Botany, University of Liverpool, P.O. Box 147, Liverpool \\ AND A. P. J. TRINCI \\ Department of Microbiology, Queen Elizabeth College, Campden Hill, London W8 7 AH
}

(Received 16 August 1978)

\begin{abstract}
A mathematical model for hyphal growth and branching is described which relates cytological events within hyphae to mycelial growth kinetics. Essentially the model quantifies qualitative theories of hyphal growth in which it is proposed that vesicles containing wall precursors and/or enzymes required for wall synthesis are generated at a constant rate throughout a mycelium and travel to the tips of hyphae where they fuse with the plasma membrane, liberating their contents into the wall and increasing the surface area of the hypha to give elongation. The hypothesis that there is a duplication cycle in hyphae which is equivalent to the cell cycle observed in unicellular micro-organisms is also included in the model. Predictions from the model are compared with experimentally observed growth kinetics of mycelia of Geotrichum candidum and Aspergillus nidulans.

The finite difference model which was constructed is capable of predicting changes in hyphal length and in the number and positions of branches and septa on the basis of changes in vesicle and nuclear concentration. Predictions were obtained using the model which were in good agreement with experimentally observed data.
\end{abstract}

\section{INTRODUCTION}

A fungal spore germinates on a solid substrate to form a mycelium which increases in length exponentially. The duration of this exponential phase varies but in a sparsely branched species it may persist until the mycelium has a total length of at least $15 \mathrm{~mm}$ (Trinci, 1974). As a mycelium increases in biomass its rate of growth decelerates from its original exponential rate and it slowly differentiates into a mature colony (Yanagita \& Kogane, 1963) which then spreads across the surface of the substrate at a linear rate.

Several basic mechanisms contribute to the formation of a mycelium. Firstly, growth is highly polarized and hyphae only extend at their tips; this feature enables hyphae to attain very high extension rates, since a long length of hypha (the peripheral growth zone) contributes to the growth of a short region (the extension zone) at its tip (Trinci, 1971). Secondly, the frequency of branch initiation is carefully regulated (Trinci, 1974). Thirdly, mechanisms such as autotropism (Robinson, 1973) regulate the spatial distribution of hyphae within the mycelium.

In this communication we present a model which describes hyphal growth and branching during the exponential phase of mycelial growth. We decided to model this phase because exponential growth is much less complex than colony growth; one region of a colony grows at the organism's maximum specific growth rate (Trinci, 1971) whilst at the same time other regions increase in biomass very slowly or may even autolyse. In addition, we believe that

* Present address: Department of Microbiology, Marischal College, The University, Aberdeen AB9 1AS. 
the formulation of a successful model for exponential growth is an essential prerequisite for modelling colony growth. Also, exponential growth proceeds indefinitely in chemostat cultures and for most of the duration of batch cultures in liquid medium (Trinci, 1971) and therefore the model could be applied to these situations.

There have been several approaches to the theoretical modelling of branching in biological systems. Leopold (1971) postulated that as a general rule a branching system adopts a form which tends to minimize the total length of the system. She showed that in certain systems branch length and number increase logarithmically with the order number of the branch. Gull (1975) has demonstrated this logarithmic relationship in mycelia of Thamnidium elegans and the branch and length ratios which he obtained are similar to those observed by Leopold for trees and a river tributary system, suggesting an efficiency of branching common to all.

A generalized model for generation of branching patterns with direct applicability to mycelial growth was described by Cohen (1967). He considered growth on a solid surface as consisting of 'local density fields' of different intensity and he obtained realistic branching patterns by applying growth and branching rules; this model also incorporates an element which simulates autotropism.

Alberghina \& Martegani (1976) have developed a model which describes exponential and transient growth in Neurospora crassa; this model incorporates various aspects of protein, ribosome and DNA synthesis and provides realistic simulations of macromolecular changes during exponential growth.

Koch (1975) has used the logistic equation to model the growth of mature colonies. He assumed that the decrease in the overall growth rate of a colony was associated with an increase in hyphal density. Koch fitted this model to the data of Deppe (1973) on twodimensional growth of colonies and to that of Trinci (1970) on three-dimensional growth of pellets of Aspergillus nidulans.

However, none of the above models considers the fundamental cellular mechanisms involved in hyphal growth. We have constructed a model which considers such mechanisms as proposed by Bartnicki-Garcia (1973) and Collinge \& Trinci (1974). Thus, vesicles, which contain wall precursors and/or the enzymes required for the insertion of the precursors into the wall, are formed throughout the mycelium. They travel towards the tips of hyphae where they fuse giving elongation. In this hypothesis it is assumed that the protoplasmic membrane of a hypha only increases in surface area as a result of such fusions. The hypothesis that there is a duplication cycle in hyphae which is equivalent to the cell cycle observed in unicellular micro-organisms (Trinci, 1978a) is also included in the model.

\section{THE MODEL}

The complexity resulting from the above hypothesis and the desire to model discrete events such as branch initiation and septation required the construction of a finite difference model. This necessarily involves approximations not found in exact solutions of differential equations but the resultant errors can be minimal and do not constitute a serious problem.

The hypha and vesicle production. The model considers a hypha to consist of tip and hyphal segments and apical and intercalary compartments (Fig. 1). Segments have no basis in reality and only serve to model the system. Intercalary compartments (regions bounded by septa) and apical compartments are actually observed in hyphae. Hyphal segments have a constant length, $l_{\mathrm{h}}$; the values chosen for Geotrichum candidum and Aspergillus nidulans were 40 and $10 \mu \mathrm{m}$, respectively. Membrane-bound vesicles are produced in hyphal segments at a constant rate $\left(R_{\mathrm{p}}\right)$ and then travel towards the tip. Vesicles are only absorbed in hyphal segments when a lateral branch is initiated behind a septum.

Vesicle transport and absorption and hyphal extension. Tip segments are initially of 




Fig. 1. Division of a hypha into hyphal and tip segments and apical and intercalary compartments.

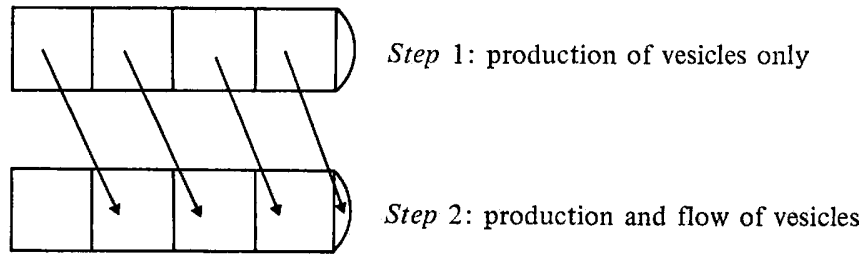

Fig. 2. Steps involved in vesicle production and flow.

negligible length and do not produce vesicles but do absorb them. The number of vesicles absorbed $\left(R_{\vee}\right)$ in the time interval, $\delta t$, is given by

$$
R_{\mathrm{v}}=\frac{R_{\mathrm{a}} \delta t V_{0}}{V_{0}+K_{\mathrm{v}}}
$$

where $R_{\mathrm{a}}$ is the maximum rate of absorption, $V_{0}$ is the number of vesicles in the tip segment and $K_{\mathrm{v}}$ is the 'saturation constant' for absorption and equals the vesicle concentration at which absorption occurs at half the maximum rate (i.e. $R_{a} / 2$ ). Thus, at low concentrations vesicle absorption by the tip is directly proportional to vesicle concentration whilst at high concentrations vesicle absorption attains a maximum rate. Absorption of vesicles during the time interval, $\delta t$, results in elongation $\left(E_{1}\right)$ of the tip segment. Thus

$$
E_{1}=\left(V_{0(t-\delta t)}-V_{0(t)}\right) R_{\mathrm{l}}
$$

where $V_{0(t)}$ is the vesicle concentration at time $t, V_{0(t-\delta t)}$ is the vesicle concentration at time $t-\delta t$ and $R_{1}$ is the increase in length due to the absorption of a single vesicle. The tip segment extends in this fashion until its length is $l_{\mathrm{h}}$ at which point a new hyphal segment is formed and the tip segment returns to having negligible length. The process is then repeated.

Flow of vesicles along the hypha is achieved by transferring vesicles from one hyphal segment to the adjacent proximal segment (hyphal or tip). This is achieved in two steps (Fig. 2). The first equation for each step illustrates the change in vesicle concentration in a hyphal segment (no. 2) and the second equation describes changes in the tip segment.

$$
\begin{array}{ll}
\text { Step 1: } & V_{2(t)}=V_{2(t-\delta t)}+\left(R_{\mathrm{p}} \times \delta t\right) \\
& V_{0(t)}=V_{0(t-\delta t)}-\frac{R_{\mathrm{a}} \times \delta t \times V_{0(t-\delta t)}}{V_{0(t-\delta t)}+K_{\mathrm{v}}} \\
\text { Step 2: } & V_{2(t)}=V_{3(t-\delta t)}+\left(R_{\mathrm{p}} \times \delta t\right) \\
& V_{0(t)}=V_{0(t-\delta t)}-\frac{R_{\mathrm{a}} \times \delta t \times V_{0(t-\delta t)}}{V_{0(t-\delta t)}+K_{\mathrm{v}}}+V_{1(t-\delta t)}
\end{array}
$$

In the first step there is no flow and accumulation and depletion of vesicles occur in the hyphal and tip segments, respectively. In the second step flow occurs from hyphal segments to adjacent proximal segments, the tip segment being supplied with vesicles from the first hyphal segment. The rate of flow of vesicles along a hypha can be increased or decreased by decreasing or increasing the ratio of steps 1 to steps 2 . 
Septation. Intercalary compartments are delimited by septa. They vary in length and consist of several hyphal segments. During septation the proportion of vesicles flowing between hyphal segments separated by a developing septum decreases at a linear rate. 'Complete' septa (i.e. septa which lack pores) or 'plugged' septa (i.e. septa whose central pores have been plugged) do not allow the passage of vesicles to the next proximal intercalary compartment. However, septa with unplugged pores allow a certain proportion of the vesicles from one intercalary compartment to flow to the next proximal compartment.

Duplication cycle. A duplication cycle (Trinci, 1978a) has also been incorporated into the model. Initially an apical compartment contains four nuclei. When the cytoplasmic volume:nuclear number ratio reaches a critical level these nuclei increase continuously and exponentially to eight. A septum is then formed midway in the apical compartment separating the nuclei equally. This duplication cycle thus regulates the frequency and site of septation.

Branching. Branch initiation occurs in the model when the flow of vesicles into a hyphal or tip segment results in attainment of a critical level of vesicles. For a tip segment this happens when the rate at which vesicle flow into the tip segment exceeds $R_{\mathrm{a}}$, the maximum rate at which they can be absorbed by the tip, and results in the formation of an apical branch. For a hyphal segment behind a septum the initiation of a lateral branch occurs because the septum prevents or reduces the flow of vesicles from the compartment.

Branch initiation in either case results in the formation of new tip and hyphal segments from the parent segment (segment of accumulation). Growth of the branch is as described above except that a proportion of vesicles flows from the parent compartment into the branch.

Spore germination. Spore germination is modelled as a means of initiating the whole process. A spore consists of a hyphal segment which produces vesicles. Germination consists in the formation of a tip segment and occurs when the number of vesicles in the spore reaches a certain level. The germ tube thus formed may be considered to be a branch from the spore.

\section{SIMULATION}

The model was simulated on a 1906S ICL digital computer with results displayed graphically on a Calcomp Graph Plotter. This involved calculations of vesicle and nuclei numbers in all segments over a series of time intervals. The various parameters required for the simulation were calculated as described below.

The maximum rate of absorption of vesicles $\left(R_{\mathrm{a}}\right)$ by tip segments was calculated from experimental data by dividing the rate of increase in protoplasmic surface area during linear growth by vesicle surface area; vesicle diameters (116 and $120 \mathrm{~nm}$ for Geotrichum candidum and Aspergillus nidulans, respectively) were estimated from experimentally observed values (Trinci \& Collinge, 1975, for G. candidum; McClure et al., 1968, for $A$. nidulans).

The increase in hyphal length $\left(R_{1}\right)$ resulting from the absorption of a single vesicle was calculated by assuming the hypha to be cylindrical; a hyphal diameter of $3 \mu \mathrm{m}$ was assumed for each species. Thus the increase in surface area $\left(A_{\mathrm{h}}\right)$ of the hypha is given by

$$
A_{\mathrm{h}}=2 \pi r_{\mathrm{h}} \delta l
$$

where $r_{\mathrm{h}}$ is the hyphal radius and $\delta l$ is the increase in hyphal length. The vesicle surface area $\left(A_{\mathrm{v}}\right)$ is given by

$$
A_{\nabla}=4 \pi r_{\mathrm{v}}^{2}
$$

where $r_{v}$ is the vesicle radius. Therefore

$$
R_{\mathrm{l}}=\delta l=\frac{4 \pi r_{\mathrm{v}}^{2}}{2 \pi r_{\mathrm{h}}}=\frac{2 r_{\mathrm{v}}^{2}}{r_{\mathrm{h}}}
$$


Table 1. Parameter values for model simulation for Geotrichum candidum and Aspergillus nidulans

$\begin{array}{lcc}\text { Parameter } & \text { G. candidum } & \text { A. nidulans } \\ \text { Maximum rate of absorption, } R_{\mathrm{a}}\left(\text { vesicles } \min ^{-1}\right) & 490 & 442 \\ \text { Unit increase in hyphal length, } R_{\mathrm{l}}(\mu \mathrm{m}) & 0.0048 & 0.0048 \\ \text { Rate of vesicle production per unit length }\left(\text { vesicles } \min ^{-1} \mu \mathrm{m}^{-1}\right) & 1 \cdot 8 & 1 \cdot 1 \\ \text { Specific rate of nuclear increase, } R_{\mathrm{n}}\left(\mathrm{min}^{-1}\right) & 0.07 & 0.07 \\ \text { Vesicle flow rate }\left(\mu \mathrm{m} \mathrm{min}^{-1}\right) & 4 & 1 \\ \text { Saturation constant for vesicle absorption, } K_{\mathrm{v}} \text { (vesicles) } & 200 & 200 \\ \text { Vesicle concentration at which branch initiation occurs, } & 1000 & 400 \\ V_{\max } \text { (vesicles) } & & \end{array}$

The rate of vesicle production per unit length of hypha $\left(R_{\mathrm{p}}\right)$ was calculated using $R_{\mathrm{a}}$ and the experimentally determined peripheral growth zone length $(w)$ of the fungus. Thus

$$
R_{\mathrm{p}}=R_{\mathrm{a}} / w
$$

Peripheral growth zone lengths (300 and $400 \mu \mathrm{m}$ for G. candidum and A. nidulans, respectively) were estimated from experimentally determined values (Fiddy \& Trinci, 1976a, $b$ ). The specific rate of nuclear increase $\left(R_{\mathrm{n}}\right)$ was obtained directly from experimental data (Fiddy \& Trinci, 1976a, b). Flow rate values for vesicles have not been determined experimentally. However, Jennings et al. (1974) quote figures for movement of nuclei and for cytoplasmic streaming in the range 0.5 to $1.0 \mathrm{~mm} \mathrm{~h}^{-1}$ and values of this order have been used for vesicle flow rate. $K_{\mathrm{v}}$ and $V_{\max }$, the vesicle concentration at which a branch is initiated, were estimated as no experimental data are available.

The proportion of vesicles allowed to flow (through septal pores) between two adjacent hyphal segments separated by an unplugged septum was 0.004 for $G$. candidum and $0 \cdot 1$ for $A$. nidulans. The same values were used to represent the proportion of vesicles flowing into a branch from its parent compartment.

Values for the above parameters calculated for $G$. candidum and $A$. nidulans are given in Table 1. Simulation of the growth of a mycelium of $G$. candidum required modification of the model as septa are formed approximately two-thirds of the distance from the tip rather than midway in the apical compartment (Fiddy \& Trinci, 1976b). Similarly A. nidulans forms three septa per cycle in the rear half of the apical compartment (Fiddy \& Trinci, 1976a). Also sub-apical branching only was modelled as apical branches are rarely observed during early exponential growth of these species.

\section{RESULTS}

\section{Geotrichum candidum}

The increase in the length of a mycelium of $G$. candidum and its branches, as predicted by the model, is shown in Fig. 3. The mycelium grows exponentially (Figs 3, 4). The germ tube hypha and branches initially grow exponentially but eventually they all attain a common linear growth rate (Fig. 3). All these features are observed experimentally and the predicted linear growth rate approximately equals that from experimental data (Table 2). The simulation shows that a branch is initiated before the germ tube hypha attains its linear growth rate and that branches attain their linear growth rates more rapidly than the germ tube hypha (Fig. 3). Both these features are observed experimentally (Plomley, 1959; Fiddy \& Trinci, 1976 b) in germ tube and branch hypha. There is a slight reduction in the rate of increase of mycelial length prior to formation of the first branch which is followed by an acceleration and a return to a constant specific rate of increase. This corresponds to the transition, in the germ tube hypha, from exponential to linear growth which is compensated for, in terms of total mycelial length, by branch formation. 




Fig. 3. Simulated growth of a mycelium of Geotrichum candidum and its individual branches: + , total mycelial length; $\diamond$, branch length.

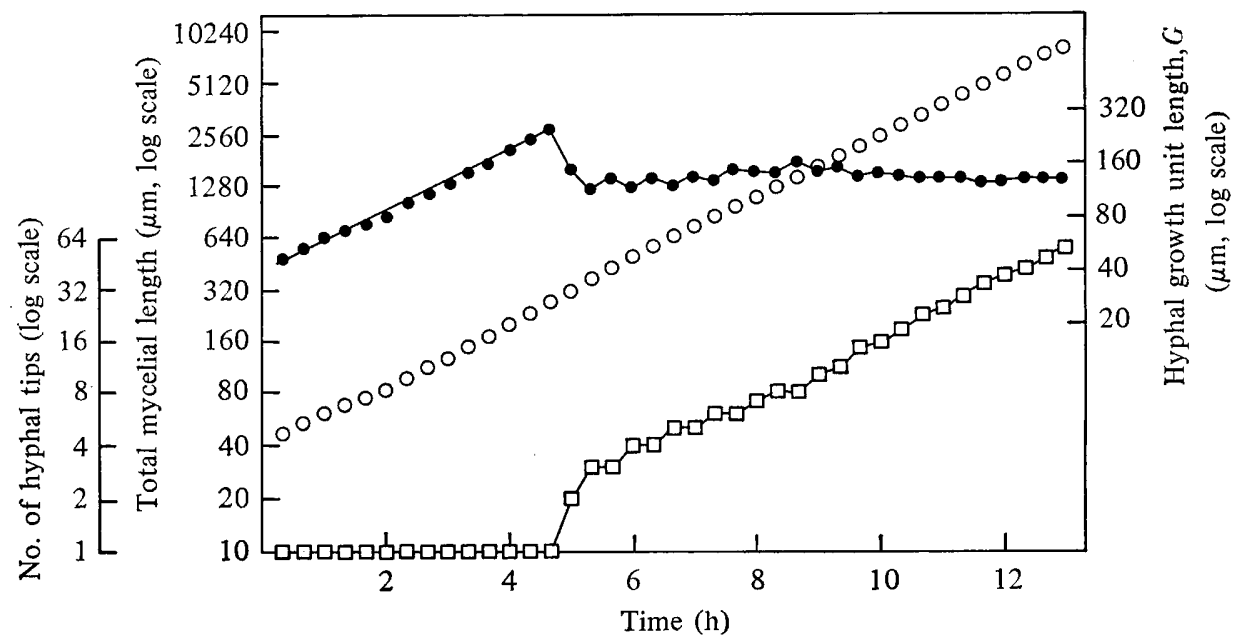

Fig. 4. Simulated growth of a mycelium of Geotrichum candidum: $\square$, number of tips; $\bigcirc$, total mycelial length; 9 , hyphal growth unit length. Compare with Fig. 1 of Trinci (1974).

Table 2. Experimental and predicted growth parameters for Geotrichum candidum and Aspergillus nidulans

G. candidum

$\overbrace{\text { Experimental* }}^{*}$ Predicted Experimental* Predicted

Parameter

Specific rate of increase in total mycelial length $\left(\mathrm{h}^{-1}\right)$

Specific rate of increase in number of branches $\left(\mathrm{h}^{-1}\right)$

Linear growth rate $\left(\mathrm{mm} \mathrm{h}^{-1}\right)$

Final hyphal growth unit length, $G(\mu \mathrm{m})$

Mean hyphal extension rate, $E \dagger\left(\mu \mathrm{m} \mathrm{h}^{-1}\right)$

Experimental values from Trinci (1974).

$\uparrow$ Determined from $E=2\left(H_{t}-H_{0}\right) /\left(B_{0}+B_{t}\right)$, where $H_{0}$ is the mycelial length in $\mu \mathrm{m}$ at zero time, $H_{t}$ is the mycelial length $1 \mathrm{~h}$ later, $B_{0}$ is the number of tips at zero time and $B_{t}$ is the number of tips $1 \mathrm{~h}$ later. The predicted values are the mean $\mathrm{cf}$ six determinations made successively at $1 \mathrm{~h}$ intervals. 


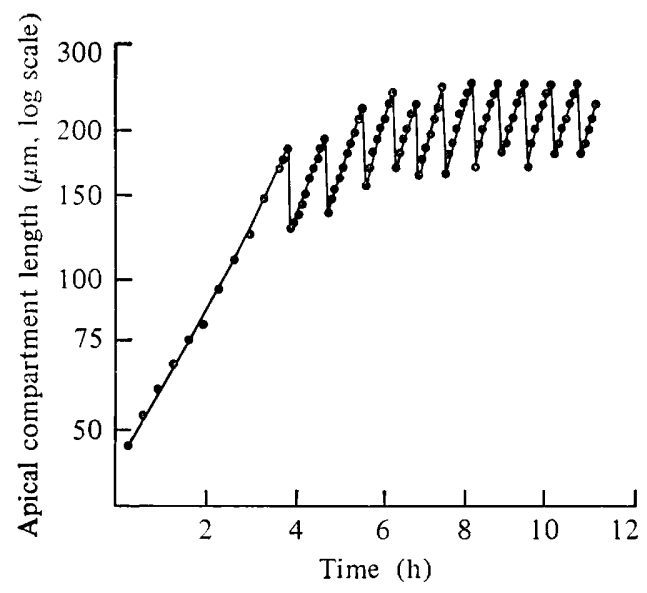

Fig. 5. Simulated growth of successive apical compartments formed by a germ tube hypha of Geotrichum candidum. Compare with Figs $3 b$ and 4 of Fiddy \& Trinci (1976b).

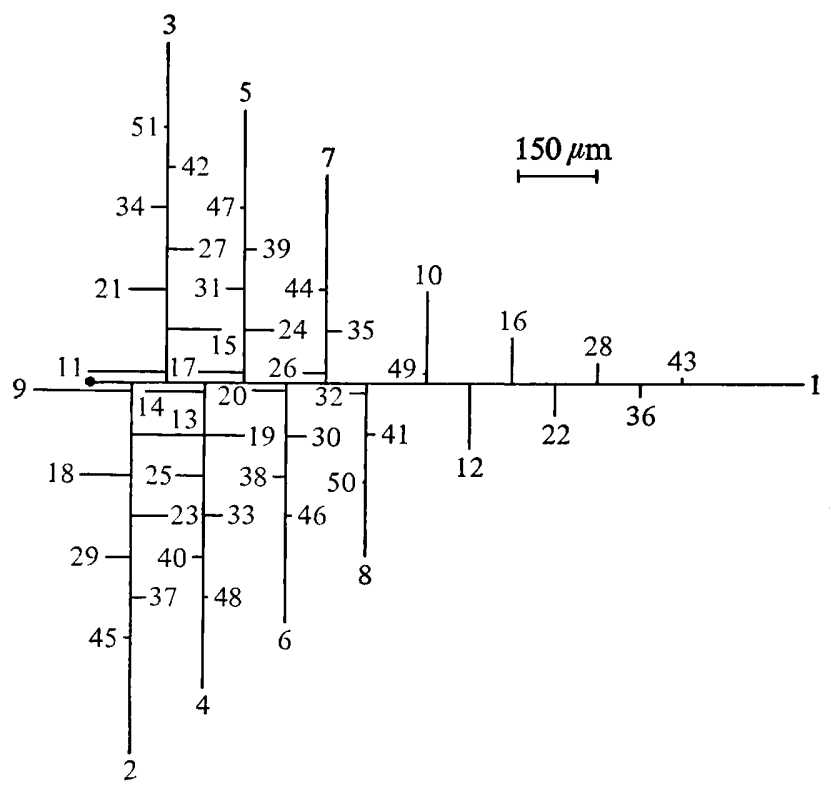

Fig. 6. Simulated morphology of a mycelium of Geotrichum candidum. Compare with Fig. $5 b$ of Trinci (1974). In every case a branch was formed in the hyphal segment behind a septum and therefore the location of branches gives the approximate position of septa. Branches are numbered in the order in which they were produced.

The number of branches in the mycelium increases exponentially at approximately the same rate as the increase in mycelial length (Fig. 4). Thus, as with experimental data (Trinci, 1974), the ratio of the mycelial length to the number of branches (i.e. $G$, the hyphal growth unit length of the mycelium) eventually tends towards a steady state value (Fig. 4). The simulation and experimental data show similar patterns for the oscillations which precede the attainment of the steady state value for $G$ and predicted and experimental values show good agreement (Table 2). In the model, as observed experimentally, mycelial length, number of branches (Table 2), number of septa and number of nuclei (not in Table) all increase exponentially at similar rates. The length of successive apical compartments formed by a germ tube of $G$. candidum is shown in Fig. 5. The apical compartment gradually 


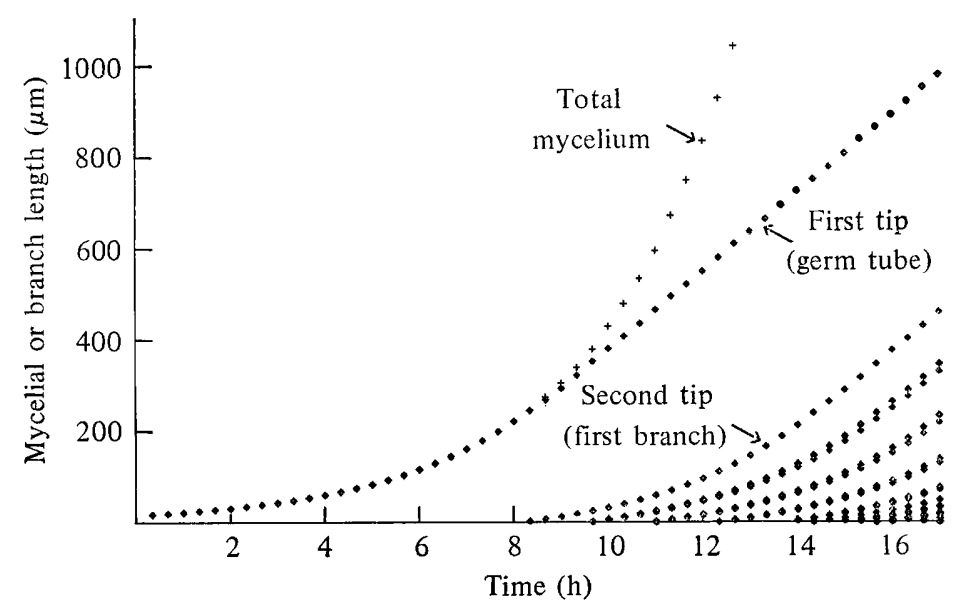

Fig. 7. Simulated growth of a mycelium of Aspergillus nidulans and its individual branches: + , total mycelial length; $>$, branch length.

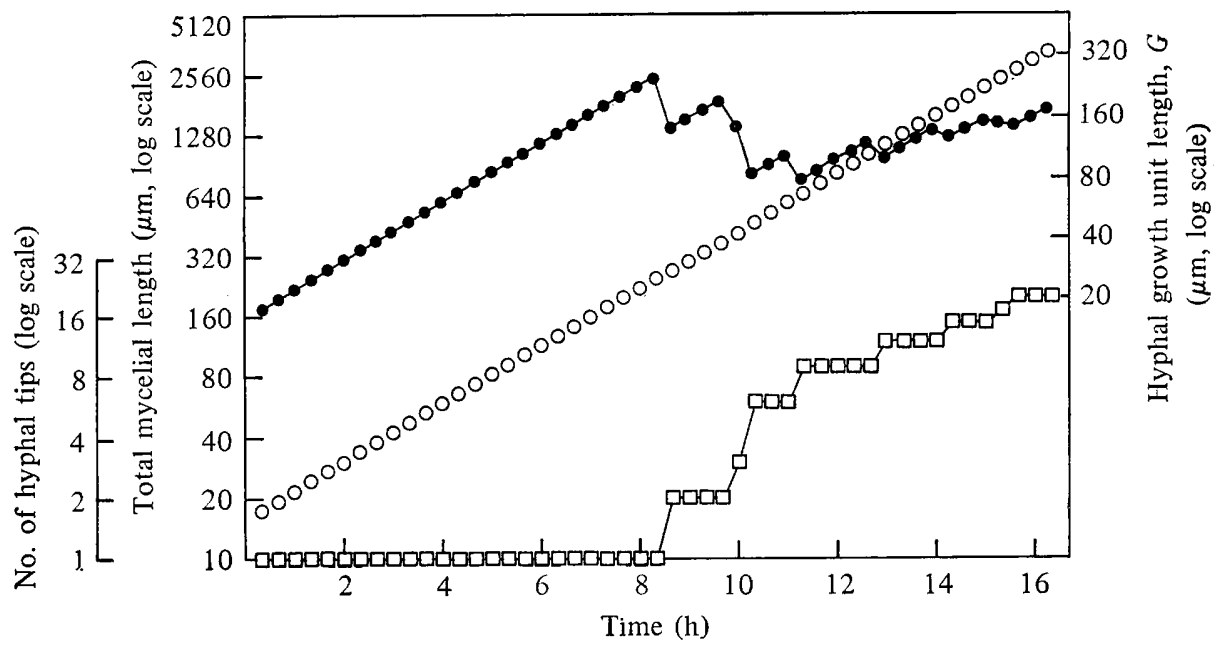

Fig. 8. Simulated growth of a mycelium of Aspergillus nidulans: $\square$, number of tips; $\bigcirc$, total mycelial length; 0 , hyphal growth unit length. Compare with Fig. 2 of Trinci (1974).

increases in length after successive periods of septation but eventually exhibits steady oscillations. Similar oscillations were observed experimentally (Fiddy \& Trinci, 1976b).

The branching pattern predicted by the model is shown in Fig. 6, assuming a branch angle of $90^{\circ}$ and branching occurring on alternate sides of the parent hypha. As observed experimentally, the branches are formed behind septa and each intercalary compartment produced one branch.

\section{Aspergillus nidulans}

Model predictions for mycelial growth of $A$. nidulans, using the parameter values in Table 1, are shown in Figs 7 and 8 and the predicted morphology of the mycelium is shown in Fig. 9. Experimental and predicted growth parameters for $A$. nidulans are compared in Table 2.

The general features of mycelial growth of $A$. nidulans are similar to those for $G$. candidum and are consistent with those observed experimentally. The total mycelial length and number of branches increase exponentially while individual hyphae show initial exponential 




Fig. 9. Simulated morphology of a mycelium of Aspergillus nidulans. Compare with Fig. $5 c$ of Trinci (1974). In every case a branch was formed in the hyphal segment behind a septum and therefore the location of branches gives the approximate position of septa. Branches are numbered in the order in which they were produced.

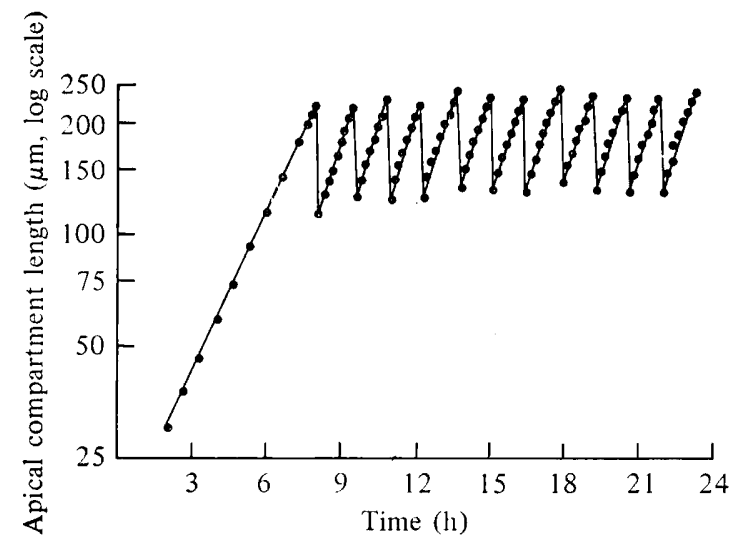

Fig. 10. Simulated growth of successive apical compartments formed by a germ tube hypha of Aspergillus nidulans.

growth followed by linear growth (Figs 7 and 8). Aspergillus nidulans can be seen to have fewer secondary branches than G. candidum (Figs 6 and 9).

Figure 10 shows the growth of successive apical compartments formed by a germ tube hypha of $A$. nidulans. As observed experimentally (Fiddy \& Trinci, 1976a) there is a gradual increase in apical compartment length during successive septation (duplication) cycles until eventually a 'steady state' oscillation in apical compartment length is attained.

Figure 11 shows the distribution of vesicles in a germ tube hypha of $A$. nidulans which is composed of an apical compartment and several intercalary compartments. There is a decrease in vesicle number in successive intercalary compartments with distance from the hyphal tip and a decrease in each compartment with distance from the tip. However, vesicles accumulate (the peaks) in hyphal segments behind septa where lateral branches may be initiated. The percentage of hyphal volume occupied by vesicles decreased in the apical compartment shown in Fig. 11 from about $0.45 \%$ in the hyphal segment behind the tip to about $0.2 \%$ in the hyphal segment adjacent to the septum. Thus, as predicted (Trinci, 


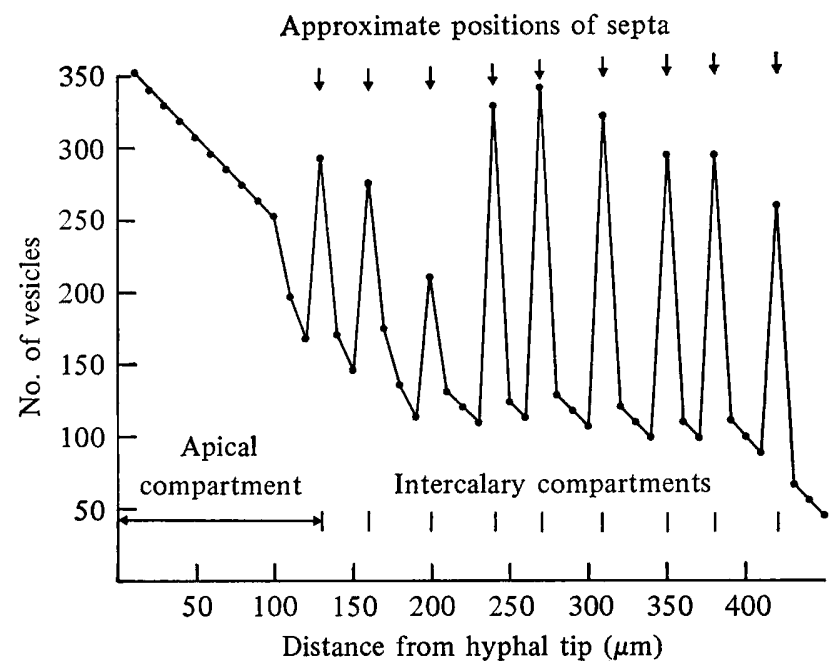

Fig. 11. Simulated distribution of vesicles along a germ tube hypha of Aspergillus nidulans which is composed of an apical compartment and several intercalary compartments.

Table 3. Effect on hyphal growth unit length of varying the rates of vesicle flow, production $\left(R_{\mathrm{p}}\right)$ and absorption $\left(R_{\mathrm{a}}\right)$

Parameter changes

Control, using the values shown in Table 1

$R_{\mathrm{p}} \times 2, R_{\mathrm{a}} \times 2$

Flow rate $\times 2$

Flow rate $/ 2$

\begin{tabular}{|c|c|}
\hline \multicolumn{2}{|c|}{$\begin{array}{l}\text { Predicted hyphal growth } \\
\text { unit length, } G(\mu \mathrm{m})\end{array}$} \\
\hline G. candidum & A. nidulans \\
\hline 140 & 115 \\
\hline 142 & 95 \\
\hline 165 & 193 \\
\hline 142 & 106 \\
\hline
\end{tabular}

$1978 b$ ), vesicles occupy only a relatively small percentage of the volume of the peripheral growth zone of a hypha. In Neurospora crassa (Collinge \& Trinci, 1974) and Penicillium chrysogenum (Collinge et al., 1978) vesicles make up about $5 \%$ of the volume of protoplasm entering the extension zone at the tips of hyphae, i.e. about 10 times more than the value obtained in the simulation. Although Fig. 11 shows that vesicles accumulate in hyphal segments distal to septa, nevertheless, even here their concentration never exceeds $0.5 \%$ of the volume of the segment. The value of 400 vesicles chosen as the level at which branch initiation occurs corresponds to approximately $0.5 \%$ of the hyphal volume. Such an accumulation would not necessarily be obvious in electron micrographs of sections of hyphae.

Table 3 shows the effects on hyphal growth unit length of varying some of the parameters shown in Table 1.

\section{DISCUSSION}

Bull \& Trinci (1977) suggest that the formulation of a mathematical model for a biological system ensures that descriptions and definitions of the system are rigorous and free of ambiguities. Modelling has the added advantages that it helps to focus attention on the fundamental properties of the system and enables predictions to be made under a limitless range of conditions. However, as observed by Topiwala (1973), a successful model does not necessarily validate basic assumptions made in a hypothesis since models based on alternative hypotheses may also produce realistic simulations. Nevertheless, if a given hypothesis cannot be modelled satisfactorily it is unlikely to be a valid one.

Collinge \& Trinci (1974) suggested that vesicles are synthesized throughout the peri- 
pheral growth zone of a hypha and then transported to the tip where they fuse with the protoplasmic membrane. Their hypothesis predicts a gradual increase in vesicle concentration in a hypha from the rear of its peripheral growth zone to the rear of its extension zone, followed by a steep increase within the tapered extension zone as the cross-sectional area of the tip declines rapidly. This hypothesis is only supported by indirect evidence (Trinci, 1978b). However, we show here that the hypothesis can be modelled successfully (Fig. 11). Since we have not incorporated an extension zone in our model, the simulation does not show the steep rise in vesicle concentration which is observed experimentally (Collinge \& Trinci, 1974) within the tapered portion of the hyphal tip (i.e. the extension zone). The model does of course allow predictions to be made of the concentration of vesicles which would be expected in various parts of a mycelium and such predictions could be used to test the hypothesis experimentally. Table 3 also illustrates the predictive value of the model.

Trinci (1974) suggested that branch initiation may be regulated by a system which involves the production, absorption and distribution of vesicles within the mycelium. He suggested that vesicles will initiate branches behind septa and the tips of hyphae if they accumulate in these regions and fuse with the rigidified wall of the hypha instead of being transported to existing extension zones. We have now shown that the spatial distribution of hyphae within a mycelium and the kinetics of branching can be successfully modelled using the vesicular hypothesis.

We wish to thank Dr M. Bazin for his advance and encouragement and the Natural Environment Research Council for financial support.

\section{REFERENCES}

Alberghina, L. \& Martegani, E. (1976). Steady and transitory states in cellular growth. Cybernetica 19, 229-248.

BARTNICKI-GARCIA, S. (1973). Microbial differentiation. Symposia of the Society for General Microbiology 23, 245-268.

Bull, A. T. \& Trinci, A. P. J. (1977). Physiology and metabolic control of fungal growth. Advances in Microbial Physiology 15, 1-84.

CoHen, D. (1967). Computer simulation of biological pattern generation process. Nature, London 216, 246-248.

Collinge, A. J. \& Trinci, A. P. J. (1974). Hyphal tips of wild type and spreading colonial mutants of Neurospora crassa. Archives of Microbiology 99, 353-368.

Collinge, A. J., Miles, E. A. \& Trinci, A. P. J. (1978). Ultrastructure of Penicillium chrysogenum hyphae from colonies and chemostat cultures. Transactions of British Mycological Society 70, $401-408$.

Deppe, C. S. (1973). Protein degradation in Schizophyllum commune. $\mathrm{Ph} . \mathrm{D}$. thesis, University of Harvard, U.S.A.

Fiddy, C. \& TrincI, A. P. J. (1976a). Mitosis, septation and the duplication cycle in Aspergillus nidulans. Journal of General Microbiology 97, 169-184.

Fiddy, C. \& TrincI, A. P. J. (1976b). Nuclei, septation, branching and growth of Geotrichum candidum. Journal of General Microbiology 97, 185-192.

GuLL, K. (1975). Mycelial branch patterns of
Thamnidium elegans. Transactions of the British Mycological Society 64, 321-324.

Jennings, D. H., Thornton, J. D., Galpin, M. F. J. \& CogGins, C. R. (1974). Translocation in fungi. Symposia of the Society for Experimental Biology 28, 139-156.

KoCH, A. L. (1975). The kinetics of mycelial growth. Journal of General Microbiology 89, 209-216.

LEOPOLD, L. B. (1971). Trees and streams: the efficiency of branching patterns. Journal of Theoretical Biology 31, 339-354.

McClure, W. K., Park, D. \& Robinson, P. M. (1968). Apical organization in somatic hyphae of fungi. Journal of General Microbiology 50, 177182.

Plomley, N. J. B. (1959). Formation of the colony in the fungus Chaetomium. Australian Journal of Biological Sciences 12, 53-64.

RoBInson, P. M. (1973). Autotropism in fungal spores and hyphae. Botanical Review 39, 367-384.

Topiwala, H. H. (1973). Mathematical models in microbiology. Methods in Microbiology 8, 35-59.

TriNCI, A. P. J. (1970). Kinetics of the growth of mycelial pellets of Aspergillus nidulans. Archiv für Mikrobiologie 73, 353-367.

TRINCI, A. P. J. (1971). Influence of the peripheral growth zone on the radial growth rate of fungal colonies. Journal of General Microbiology 67, 325-344.

TrINCI, A. P. J. (1974). A study of the kinetics of hyphal extension and branch initiation of fungal mycelia. Journal of General Microbiology 81, 225-236. 
Trinci, A. P. J. \& Collinge, A. J. (1975). Hyphal wall growth in Neurospora crassa and Geotrichum candidum. Journal of General Microbiology 91, 355-367.

TrincI, A. P. J. (1978a). The duplication cycle and vegetative development in moulds. In The Filamentous Fungi, vol. III, pp. 132-163. Edited by J. E. Smith \& D. R. Berry. London: Edward Arnold.
TRINCI, A. P. J. (1978b). Wall and hyphal growth. Science Progress 65, 75-99.

Yanagita, T. \& Kogane, F. (1963). Cellular differentiation of growth mold colonies with special reference to phosphorus metabolism. Journal of General and Applied Microbiology 9, 313-330. 